\title{
An Aerial Image Recognition Framework using Discrimination and Redundancy Quality Measure
}

\author{
Yuxing Hu and Luming Zhang \\ School of Computing, National University of Singapore
}

\begin{abstract}
Aerial image categorization plays an indispensable role in remote sensing and artificial intelligence. In this paper, we propose a new aerial image categorization framework, focusing on organizing the local patches of each aerial image into multiple discriminative subgraphs. The subgraphs reflect both the geometric property and the color distribution of an aerial image. First, each aerial image is decomposed into a collection of regions in terms of their color intensities. Thereby region connected graph (RCG), which models the connection between the spatial neighboring regions, is constructed to encode the spatial context of an aerial image. Second, a subgraph mining technique is adopted to discover the frequent structures in the RCGs constructed from the training aerial images. Thereafter, a set of refined structures are selected among the frequent ones toward being highly discriminative and low redundant. Lastly, given a new aerial image, its sub-RCGs corresponding to the refined structures are extracted. They are further quantized into a discriminative vector for SVM classification. Thorough experimental results validate the effectiveness of the proposed method. In addition, the visualized mined subgraphs show that the discriminative topologies of each aerial image are discovered.
\end{abstract}

Keywords: Aerial Image, Categorization, Discriminative, Subgraph, Data mining

\section{Introduction}

Aerial image categorization is an important component for many applications in artificial intelligence and remote sensing [18, 19, 26], such as visual surveillance, navigation, and robot path planning. However, it is still a challenging task to deal with aerial 
image categorization successfully due to two reasons. On one hand, the aerial image components (e.g., house roofs and grounds) as well as their spatial configurations are complex and inconstant, making it difficult to extract features sufficiently discriminative for aerial image representation. On the other hand, the efficiency of the existing aerial image categorization methods is far from practical due to the huge number of various components as well as their bilateral relationships. Therefore, a discriminative and concise aerial image representation has become increasingly imperative for a successful categorization system.

In the literature of designing discriminative image representations for visual recognition, many features have been proposed. They can be categorized into two groups: global features and local features. Global features, such as histograms, eigenspace [1], and skeletal shape [2], generalize the entire image with a single vector and are standard for statistic models like SVM. However, global features are sensitive to occlusion and clutter. Besides, these representations typically rely on a preliminary segmentation of objects in images. These two limitations result in unstable categorization performance. Different from global features, local features are developed to increase the discrimination, such as scale invariant feature transform (SIFT) [3]. Each local feature describes a localized image region and is calculated around the interest points. Thus, they are robust to partial occlusion and clutter. To take advantage of this property, local features [11, 12, 13] (e.g., junction [4], gradient [5], contour, etc) are widely used for aerial image parsing recently. However, when employing local features for image categorization, different images typically contain different numbers of local features. That is, it is difficult to integrate the local features within an image for the standard classifiers. In many cases, they are integrated into an orderless bag-of-features as global representation, thereby the similarity between images is determined by the orderless bag-of-features. It is worth emphasizing that as a non-structural representation, the bags-of-features representation ignores the geometric property of an image (i.e., the spatial distribution of the local image patches), which prevents it from being highly discriminative. Given the zebra skin and the chessboard skin, their bag-of-features representations are similar. That is to say, the bag-of-features representation is not sufficiently descriptive to distinguish the zebra and the chessboard, although the geometric 
properties of the two images are significantly different.

In order to encode image geometric proprieties into a categorization model, several image geometric features have been proposed. In 〔14], the spatial pyramid matching kernel is obtained by clustering the local features into a few geometric types. However, the spatial pyramid matching kernel is not flexible enough, since it highly depends on the human prior knowledge. RGB-domain spin image [15] describes the spatial context by exploring the chain structure of pixels in each RGB channel. However, the chain structure usually fails to describe the spatial context with complicated structures. Walk kernel [16] is proposed to capture the walk structures among image local features. However, the unavoidable totter phenomenon (i.e., one vertex may occur several times in a walk) brings noise and hence limiting its discrimination. To obtain a better discrimination, parameters are provided to tune the length of the chain [15] or walk [16]. This operation leads to very redundant structures. Both the time consumption and the memory cost increase remarkably as the structure number goes up. Therefore, a concise image structure representation is desired for accurate aerial image categorization. Recently, many graph-based models are applied in intelligence systems and multimedia. They can be used as geometric image descriptors [38, 39, 27, 28] to enhance image categorization. Besides, these methods can be used as image high-order potential descriptors of superpixels [29, 30, 31, 32, 6]. Further, graph-based descriptors can be used as a general image aesthetic descriptors to improve image aesthetics ranking, photo retargeting and cropping [7, 8, 9, 10].

In this paper, we propose a novel aerial image categorization system, which enables the exploration of the geometric property embedded in local features. An aerial image is represented by a graph, since graph is a natural and descriptive tool to express the complicated relationships among objects. By defining region connected graph (RCG), we decompose an aerial image into a set of discriminative subgraphs. To capture discriminative relationships among RCGs, a structure refinement strategy is carried out to select highly discriminative and low redundant structures. Based on the refined structures, we extract sub-RCGs accordingly and all the sub-RCGs from an aerial image form the discriminative spatial context. Finally, a quantization operation transforms the discriminative spatial context into a feature vector for categorization. 
The major contributions of this paper are as follows: 1) region connected graph (RCG), a graph-based representation that describes the local patches and their topology for an areal image; 2) a structure refinement algorithm that selects highly discriminative and low redundant structures among the training RCGs; and 3) an efficient isomorphism subgraph extraction component that acquires the corresponding sub-RCGs.

\section{Region Connected Graph(RCG)}

An aerial image usually contains millions of pixels. If we treat each pixel as a local feature, highly computational complexity will make aerial image recognition intractable. Fortunately, an aerial image can be represented by a collection of clusters because pixels are usually highly correlated with their neighboring ones. Each cluster consists of neighboring pixels with consistent color intensities. Thus, given an aerial image, we can represent it by a set of regions instead of millions of pixels. The neighboring relationships between regions define the spatial context of an aerial image. Naturally, we can model this representation as a labeled graph. The labels denote the local features of each region and each edge connects pairwise neighboring regions. In our work, we call this representation region connected graph (RCG).

To obtain the RCG from an aerial image, a segmentation algorithm (i.e., fuzzy
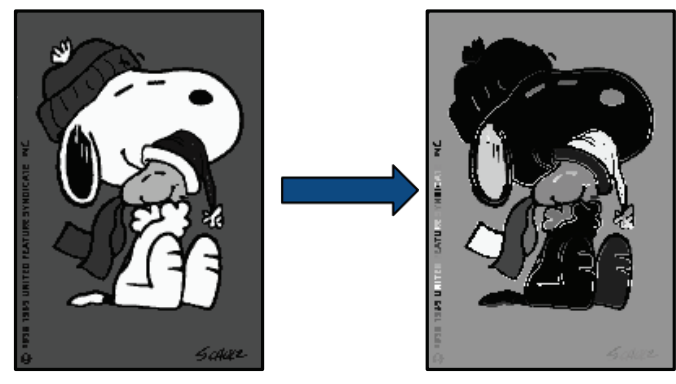

Figure 1: From pixel clusters (left) to singly connected regions (right).

clustering [37] in our implementation) groups pixels into different clusters according to their color intensity. Note that the pixels in the same cluster are unnecessarily spatially neighboring. As shown in Fig. 1, we use different grayscale values to identify different clusters. Pixels in the face and the lower half of the Snoopy's body are grouped into 
the same cluster. However, it is more reasonable if they are categorized into different groups, since the face and the lower half of Snoopy are spatially isolated. To this end, a region growing algorithm [17] is employed to divide an image into regions iteratively. In each iteration, the region growing algorithm initializes the current region with a random pixel. It continues adding the spatially neighboring pixels into this region if the current pixel and the existing pixels come from the same cluster. The iteration terminates if the entire pixels are considered. The clustering result is shown on the right of Fig. 11

On the basis of the singly connected regions, the RCG of an aerial image can be

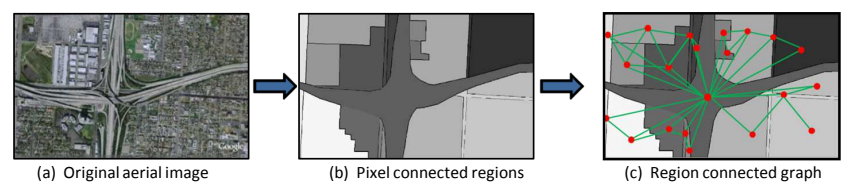

Figure 2: The flowchart from an aerial image to its RCG.

obtained as shown in Fig. 22 Given an aerial image $I$ (Fig. 22 a)), we segment it into $K$ singly connected regions $R=\left\{r^{i}\right\}_{i=1 \cdots K}$ (Fig. 2 2 (b)). Then, each singly connected region is treated as a vertex $v_{i}$ (the red solid point), and the relationship between spatially neighboring vertices is linked by an edge $e_{j}$ (the green line). Finally, denoting $V=\cup v_{i}$ as a collection vertices $v_{i}$ and $E=\cup e_{j}$ a set of edges $e_{j}$, we define $\mathcal{G}=(V, E)$ as an RCG, where $V$ is a set of singly connected regions and $E$ is a set of spatially neighboring relationships (Fig. [2 $(\mathrm{c})$ ). Let $|G|$ denote the number of vertices in RCG $G$. The number of neighbors of a vertex is called the vertex degree. A useful attribute of RCG is that its vertex degree is upper bounded. That is to say, each region has a limited number of neighbors. It is observed that the average vertex degree of each RCG is less than four and the maximum vertex degree is no more than 15 .

\section{Discriminative Structures Selection}

It is natural to recognize an aerial image by matching its RCG to a labeled one. However, as proved in [22], given a pair of graphs, it is NP-hard to determine whether they have the same structure. That means it is intractable to compare pairwise RCGs 
directly. Alternatively, we represent an aerial image by a set of sub-RCGs $\left\{G_{s u b}^{k}\right\}_{k=1 \cdots N}$, where $\cup_{k=1}^{N} G_{\text {sub }}^{k}=G$. Thereby, the aerial image categorization can be conducted by matching its sub-RCGs to those of the labeled aerial images. Noticeably, the RCG of an aerial image may contain tens to hundreds of vertices. Given $n$ vertices in an RCG, there will be $N=2^{\frac{(n+1) * n}{2}}$ different sub-RCGs, which makes it impractical to represent an aerial image by enumerating all its sub-RCGs (Fig. 3 a)). Toward a discriminative and concise representation for aerial image recognition, only sub-RCGs with highly discriminative and low redundant structures should be selected for aerial image categorization (Fig. 3 (b)).

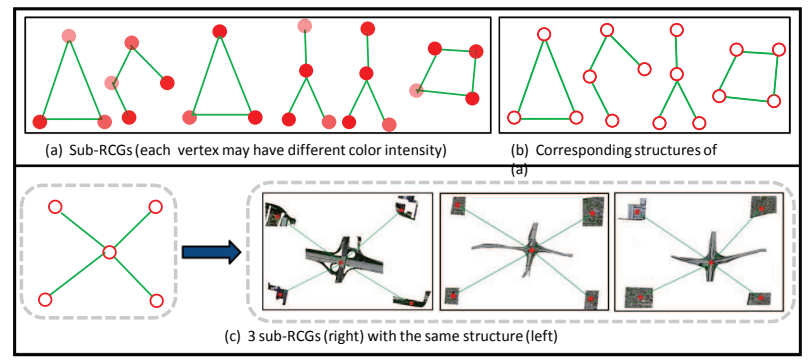

Figure 3: An example of sub-RCGs and their structures.

\subsection{Frequent Structures Mining}

Each sub-RCG reflects the structure of a subset of connected vertices in the RCG. In other words, a sub-RCG models the spatial context of an aerial image. Different types of aerial image are with different spatial context, so do the structures of subRCGs. It is natural to use the structure of sub-RCG to determine the aerial image type. For instance, as shown in Fig. 3. (c), all the three sub-RCGs share the same structure but slightly different color intensity distributions. However, it is impractical to enumerate all the possible sub-RCGs. Moreover, only those frequently occurred sub-RCGs contribute to the recognition task while the others are redundant. Motivated by these, we have to select the frequent structures.

In our implementation, an efficient frequent subgraph discovery algorithm called FSG [21] is employed. It is noticeable that the vertex value of sub-RCGs might be 
different though they share the same structure. This prevents us from mining the frequent structures accurately. Therefore, we ignore the difference of vertex values. In particular, given a sub-RCG, its structure $S$ is obtained by setting the vertex labels of the sub-RCG to a same value, e.g., one.

FSG accumulates the times of happening for each structure. It outputs the probabilities of all the structures in the training RCGs, implying that the structure is unnecessarily existing in all the training RCGs. A probability $p(S)$ represents the frequency of $S$. As the number of original candidate structures is exponential, only the structure whose probability is higher than a threshold is output as a frequent one. Therefore, the number of frequent structures is greatly reduced greatly.

\subsection{Measures for Structure Selection}

The number of frequent structures is still too large (typically 100 300) though it is much smaller than that of the candidate structures. In addition, a structure with high frequency may not be highly discriminative. Thus, we carry out a further selection among the frequent structures to preserve only the highly discriminative and low redundant ones. We first define a distance to describe the similarity between sub-RCGs $\left(G_{\text {sub }}\right.$ and $\left.G_{\text {sub }}^{\prime}\right)$ with the same size:

$$
d\left(G_{s u b}, G_{\text {sub }}^{\prime}\right)=\sum_{v_{r} \subseteq G_{s u b} \wedge v_{r}^{\prime} \subseteq G_{s u b}^{\prime}}\left\|f\left(v_{r}\right)-f\left(v_{r}^{\prime}\right)\right\|^{2}
$$

where $v_{r}$ is $r$-th vertex of $G_{s u b}$ and $f(\cdot)$ the local regions' feature vector. $\|\cdot\|$ is the Euclidean norm. More specifically, for structure $S$ and $S^{\prime}$ in $G$ and $G^{\prime}$ respectively, if $|S|=\left|S^{\prime}\right|$, we define the structure distance between $G$ and $G^{\prime}$ as follows:

$$
d_{e}\left(S_{G}, S_{\left(G^{\prime}\right)}^{\prime}\right)=\varphi \cdot \sum_{G_{s u b}(S) \subseteq G} \cdot \sum_{G_{s u b}^{\prime}\left(S^{\prime}\right) \subseteq G^{\prime}} d\left(G_{s u b}(S), G_{s u b}^{\prime}\left(S^{\prime}\right)\right)
$$

where $G_{\text {sub }}\left(S_{(\cdot)}\right)$ is the sub-RCG corresponding to $S_{(\cdot)} . \varphi$ is a factor that normalize $d_{e}$

to $[0,1]$ and it is not a tuning parameter. That is, $\varphi=\frac{1}{\left(\left|G_{s u b}\right| \cdot\left|G_{s u b}^{\prime}\right|\right.}$, where $\left|G_{\text {sub }}\right|$ and $\left|G_{\text {sub }}^{\prime}\right|$ denote the number of sub-RCGs in RCG $G$ and $G^{\prime}$, respectively. By extending Eq.(2) to the situations when $\|S\| \neq\left\|S^{\prime}\right\|$, we define a more generic form of the structure distance between $G$ and $G^{\prime}$. It is based on the probability $p(S)$ by taking into account 
of different situations.

$$
d\left(S_{G}, S_{G^{\prime}}^{\prime}\right)=\left\{\begin{array}{l}
p(S) * p\left(S^{\prime}\right) * d_{e}\left(S_{G}, S_{\left(G^{\prime}\right)}^{\prime}\right) \\
\quad \text { if }|S|=\left|S^{\prime}\right| \text { and } G_{s u b} \neq \emptyset \wedge G_{\text {sub }}^{\prime} \neq \emptyset \\
p(S) * p\left(S^{\prime}\right) * \sum_{i} d_{e}\left(S_{G}, C_{i}\left(S_{G^{\prime}}, S_{G}\right)\right) \\
\quad \text { if }|S|<\left|S^{\prime}\right| \text { and } G_{s u b} \neq \emptyset \wedge G_{s u b}^{\prime} \neq \emptyset \\
p(S) * p\left(S^{\prime}\right) * \sum_{i} d_{e}\left(S_{G}^{\prime}, C_{i}\left(S_{G}, S_{G}^{\prime}\right)\right) \\
\text { if }|S|>\left|S^{\prime}\right| \text { and } G_{s u b} \neq \emptyset \wedge G_{\text {sub }}^{\prime} \neq \emptyset \\
(1-p(S)) *\left(1-p\left(S^{\prime}\right)\right) \\
\text { if } G_{\text {sub }}=\emptyset \wedge G_{\text {sub }}^{\prime}=\emptyset \\
p(S)+p\left(S^{\prime}\right)-2 p(S) * p\left(S^{\prime}\right) \\
\text { otherwise }
\end{array}\right.
$$

The probability for structure $S$ existing in $G$ is denoted by $p(S)$. It is straightforward to obtain the first line of Eq. (3) by multiplying $p(S) * p\left(S^{\prime}\right)$ with the structure distance $d_{e}\left(S, S^{\prime}\right)$ wherein $p(S) * p\left(S^{\prime}\right)$ denotes the probability for $S$ existing in $G$ and $S_{2}$ existing in $G^{\prime}$. This is similar to the second line and the third line of Eq.(3). As $S$ is a subset of $S^{\prime}$ when $|S|<\left|S^{\prime}\right|$, the function $C\left(S, S^{\prime}\right)$ outputs the enumerated structures with the same size to $S^{\prime}$ in $S$ by FSG [21] in the second line of Eq. [3], and vice versa in the third line. $(1-p(S)) *\left(1-p\left(S^{\prime}\right)\right)$ denotes the probability for neither $S$ existing in $G$ nor $S^{\prime}$ existing in $G^{\prime}$. An $p(S)+p\left(S^{\prime}\right)-2 p(S) * p\left(S^{\prime}\right)$ in the last line is the probability for either $S$ existing in either $G$ or $S^{\prime}$ existing in $G^{\prime} . d\left(S_{G}, S_{G^{\prime}}^{\prime}\right) \in[0,1]$.

Based on the structure distance $d\left(S_{G}, S_{G^{\prime}}^{\prime}\right)$ between $G$ and $G^{\prime}$, measure of structure discrimination(MSD), is defined for structure's discrimination. Inspired by the definition of discriminative ability in LDA [25], MSD computes the distance ratio between RCGs with different labels and those with same labels:

$$
M_{s d}(S)=\frac{D_{S}^{b}}{D_{S}^{w}}=\frac{\sum_{G} \sum_{G^{\prime}} d\left(S_{G}, S_{G^{\prime}}^{\prime}\right) * \sigma}{\sum_{G} \sum_{G^{\prime}} d\left(S_{G}, S_{G^{\prime}}^{\prime}\right) * \sigma^{\prime}}
$$

$\sigma$ and $\sigma^{\prime}$ are functions indicating whether $G$ and $G^{\prime}$ are belong to the same class. If $G$ and $G^{\prime}$ belong to different classes, $\sigma=1, \sigma^{\prime}=0$, otherwise $\sigma=1, \sigma^{\prime}=\mathrm{d}^{1}$. A

\footnotetext{
${ }^{1}$ Pairwise RCGs $G$ and $G^{\prime}$ belonging to the same class means that their corresponding aerial images belong to the same class. Similarly, two RCGs $G$ and $G^{\prime}$ belonging to different classes means that their
} 
larger $M_{s d}(S)$ means a more discriminative ability of structure $S$. However, a structure set with high discrimination doesn't mean it is a concise one. Aiming at a concise set of structures, it is necessary to make further structure selection. Motivated by the fact that high correlation leads to high redundancy [23], we believe that one of the two structures should be removed if two structures are highly correlated. In order to calculate the correlation between structures, an approach to quantize the redundancy between structures, called measure of structures correlation (MSC), is defined based on the distance between structures:

$$
M_{s c}\left(S, S^{\prime}\right)=\frac{\sum_{G} \sum_{G^{\prime}} d\left(S_{G}, S_{G^{\prime}}^{\prime}\right)}{\sum_{G} \sum_{G^{\prime}} d\left(S_{G}, S_{G^{\prime}}\right)+\sum_{G} \sum_{G^{\prime}} d\left(S_{G}^{\prime}, S_{G^{\prime}}^{\prime}\right)}
$$

where the denominator functions as a normalization step. A larger $M_{s c}\left(S, S^{\prime}\right)$ leads to a lower correlation between structure $S$ and $S^{\prime}$, and vice versa. Eq.(5) also can be explained by analogy with the three vertices of a triangle in Fig. 4 $\sum_{G} \sum_{G^{\prime}} d\left(S_{G}, S_{G^{\prime}}^{\prime}\right)$, $\sum_{G} \sum_{G^{\prime}} d\left(S_{G}, S_{G^{\prime}}\right)$ and $\sum_{G} \sum_{G^{\prime}} d\left(S_{G}^{\prime}, S_{G^{\prime}}^{\prime}\right)$ act as the distance between the three vertices. When $\sum_{G} \sum_{G^{\prime}} d\left(S_{G}, S_{G^{\prime}}^{\prime}\right)$ becomes larger, the correlation between $S$ and $S^{\prime}$ becomes lower (Fig.4(c)), and vice versa (Fig. 4(b)).

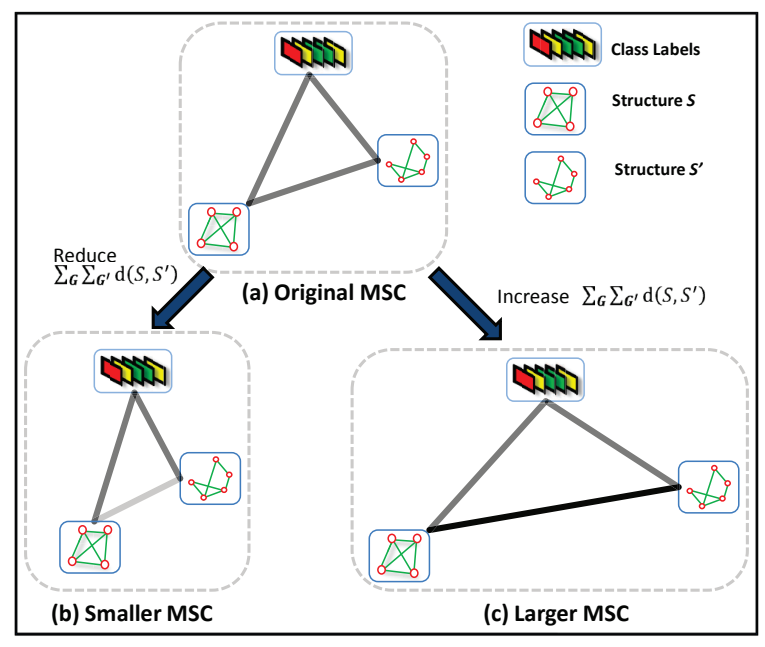

Figure 4: A visual explanation of the correlation between structures.

corresponding aerial images belong to different classes. 


\subsection{MSD and MSC based Structure Refinement}

Based on the two structure measures MSD and MSC, we construct a novel concise and discriminative structure refinement algorithm. The stepwise operations of the proposed structure selection are illustrated in and Algorithm 1 respectively. The algorithm can be divided into two steps. First, the MSD values of all the candidate structures are computed and sorted in descending order. Candidate structure whose MSD value is higher than a threshold will be preserved initially into the list $L_{\text {final }}$. Second, the MSC value between each pair of preserved structures is computed to evaluate their redundancy. The removal of redundant structures is carried out iteratively. During the first round of iteration, we specify the preserved structure with the largest MSD value as the final selected one. Then, we sort the MSC values between the finally selected structure and the rest of the preserved structures. The structure whose MSC value is higher than a threshold will be removed. The preserved structure list will be updated accordingly. After one round of iteration, we move to the preserved structure with lower MSD value. The iteration terminates when there is no structure next to $S_{\text {final }}$. The finally preserved structures are deemed as the refined ones.

Denote $n$ as the number of training RCGs and $m$ as the number of candidate structures, we assume that the structure distance between RCGs can be computed in constant time. As the distance between RCGs is required for calculating MSD and MSC, the computational cost of calculating MSD and MSC are both $O\left(n^{2}\right)$. As shown in Algorithm 1, the structure refinement step contains a double loop and the time complexity of each is $O\left(n^{2} * m\right)$. Therefore, the time complexity of the whole selection process is $O\left(n^{2} * m^{2}\right)$.

\section{Geometric Discriminative Feature}

\subsection{Geometric Discriminative Feature Extraction}

As the refined structures are both concise and discriminative, they are adopted to extract the geometric discriminative features. Guided by the refined structures, we extract sub-RCGs with the same structures and then use them as the geometric discriminative features. As RCGs are low degree graphs (vertex degree less than 15), the 


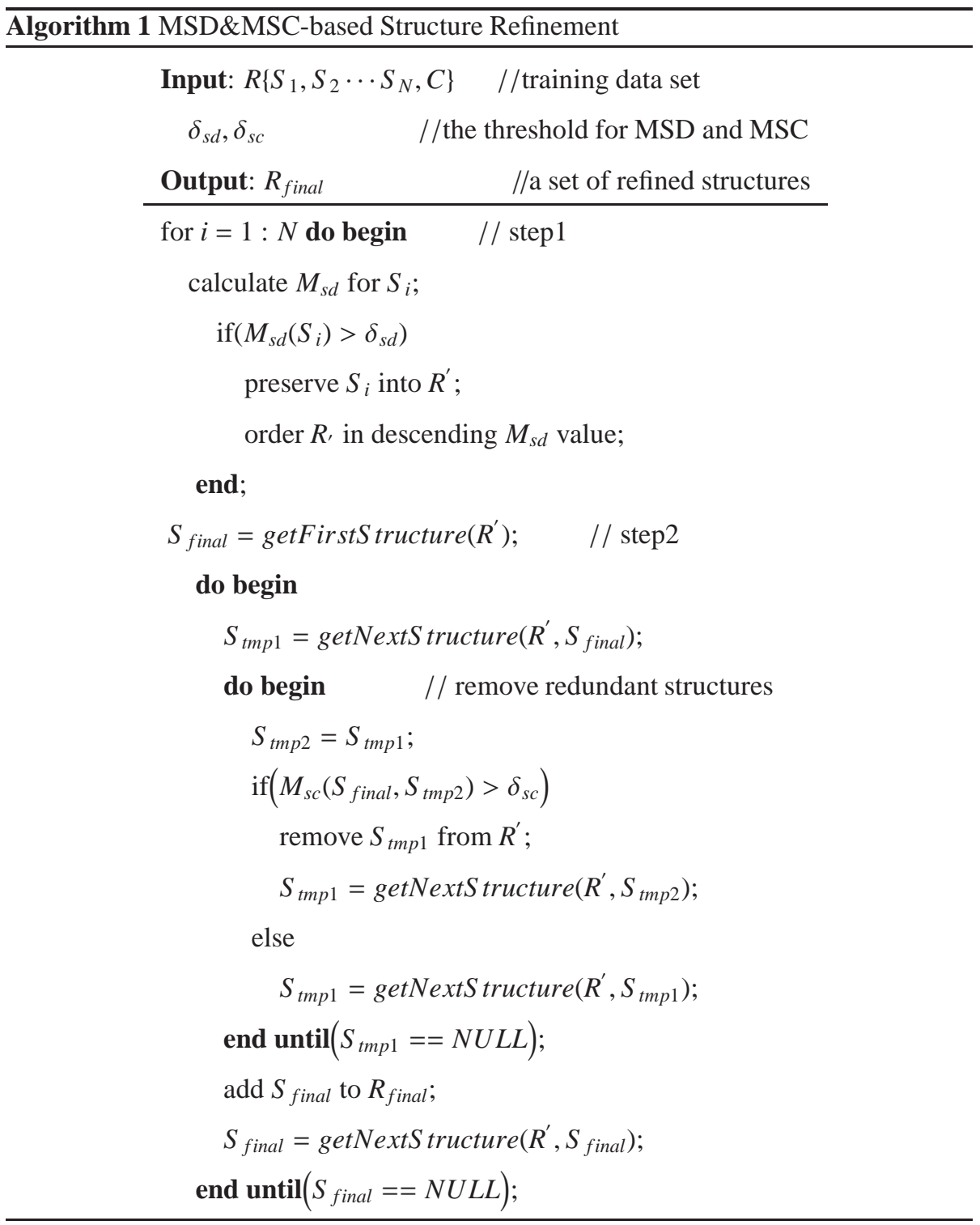


computational complexity is nearly linear increasing with the number of vertices $\lfloor 16]$.

To achieve an efficient sub-RCG extraction process, we propose an algorithm to locate the sub-RCGs efficiently. Given a refined structure $S$ and an RCG $G$, the proposed algorithm outputs a collection of sub-RCGs with structure $S$. There are three steps in the proposed geometric discriminative feature extraction. First, the vertices of $S$ are checked to determine whether $|S| \leq|G|$. If $|S| \leq|G|$, then an iterative process will be carried out. Otherwise, the algorithm will terminates. Next, for each vertex in $G$, we treat it as the reference point and compare $S$ to the structures of its correlated subRCGs. A depth-first-search strategy [40] is employed for graph matching. Only the sub-RCGs with the same structure to $S$ are the preserved. By traversing all the vertices in RCG $G$, we perform the matching process and collect all the qualified sub-RCGs. Finally, a collection of qualified sub-RCGs are obtained

\subsection{Quantizing Sub-RCGs into Feature Vectors}

Given an aerial image, it can be represented by a set of sub-RCGs as described above. It is worth emphasizing that the sub-RCGs are planar visual feature in $\mathbb{R}^{2}$. Conventional classifiers such as support vector machine (SVM) [24] can only handle 1-D vectors. Further, the number the extracted sub-RCGs are different from one aerial image to another. Therefore, it is impractical for a conventional classifier like SVM to carry out classification directly. To tackle this problem, a quantization method is developed to convert each aerial image into a 1-D vector.

The proposed quantization method is based on the distances between the test aerial

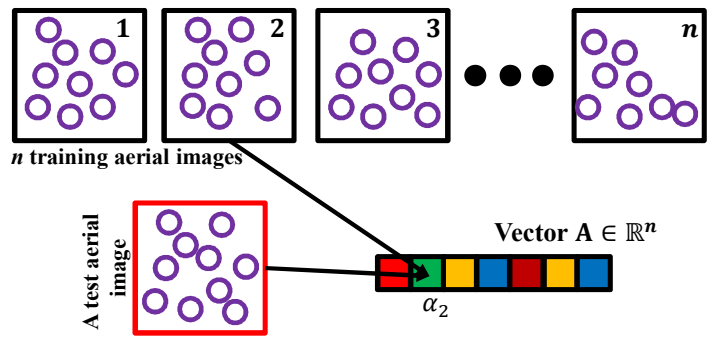

Figure 5: An illustration of generating the feature vector for a test aerial image. The blue circles in each aerial image denote the sub-RCGs. 
images and the training ones. The distance is computed using the extracted geometric discriminative features. Given an aerial image, we first extract its geometric discriminative features, each corresponding to a refined structure. Then. as shown in Fig. 5, an aerial image is encoded into a vector $\mathbf{A}=\left[\alpha_{1}, \alpha_{2} \cdots, \alpha_{n}\right]^{T}$, where $n$ is the number of training aerial images and each element of $\alpha$ is computed as:

$$
\alpha_{i} \propto \exp \left(-\lambda * \sum_{S} d\left(S_{G}, S_{G^{\prime}}\right)\right)
$$

where $\lambda$ is a free parameter to be tuned. In our implementation, we fix $\lambda$ to 0.5 by using cross validation.

\section{System Overview}

Our aerial image categorization system can be divided into the training and the test stages. In the training phase, structure refinement for geometric discriminative feature extraction is conducted. First, each aerial image is segmented into connected regions for building the corresponding RCGs. Then, a frequent structure mining algorithm is employed to discover the highly frequent structures in the training RCGs. Next, MSD and MSC are computed for each structure toward a concise set of structures. Structure refinement is carried out to acquire the highly discriminative and low redundant ones. Third, the geometric discriminative features are obtained by extracting the subRCGs corresponding to the refined structures. To convert the extracted 2-D geometric discriminative features into 1-D vectors, a quantization scheme computes the distance between the given aerial image and the training samples. Finally, we train an SVM classifier by the vectors from the encoded training samples.

The test phase is illustrated on the right. Given a test aerial image, we obtain its RCG firstly. Then, the geometric discriminative features are extracted to represent the given aerial image. Similarly, a quantization operation is carried out to convert the

aerial image into a vector using the geometric discriminative features. This vector is fed into the trained SVM for aerial image categorization. 


\section{Experiments and Results Analysis}

Experiments are carried out on two data sets. The first data set contains the aerial images from the Lotus Hill (LHI) data set [36]. It consists of five categories where each category contains 20 aerial images. Each image is associated with a standard segmentation map. The second data set is our own complied data set and it includes aerial images from ten categories. The whole data set contains 2,096 aerial images crawled from the Google Earth. The experimental system is equipped with an Intel E8500 CPU and 4GB RAM. All the algorithms are implemented on the Matlab platform.

\subsection{Comparative Study}

In our experiment, the validation of the proposed geometric discriminative feature is conducted on both the LHI and our own data sets. We compare our geometric discriminative feature with several representative discriminative visual features, i.e., the global RGB histogram, the intensity-domain spin images [15], the walk/tree kernel [16], the sparse coding spatial pyramid matching (SC-SPM) [42], the localityconstrained spatial pyramid matching (LLC-SPM) [41], and the object bank [43]. As the spatial pyramid matching kernel [14] heavily relies on the prior knowledge, we do not employ it for comparison. In our implementation, the geometric discriminative features are extracted to encode both the color intensity distribution and the spatial property. In each segmented region, a 4096-dimensional RCB-histogram is extracted as its representation. A few example aerial images and their geometric discriminative features are presented.

First, we present a set of discovered discriminative subgraphs. From a horizontal glance, we can roughly discriminate aerial images from the five categories, especially for the intersections and the marines. This demonstrates the necessity to exploit the relationships among aerial image patches for categorization.

Further, to make comparison among the global histogram, the spin images, the walk kernel, and the proposed geometric discriminative feature, we select half of the images for training and leave the rest for testing. As shown in Table 1 , the proposed feature achieves the best accuracy on average. 
Table 1: Recognition rate with standard deviation on our own data set(the experiment was repeated 10 times; $\mathrm{HC}$ is the HOG+color moment with a 1024-sized codebook; the number in each bracket denotes the codebook size; and LR2 and LRG are different regularizers as described in [43])

\begin{tabular}{|c|c|c|c|c|c|c|c|c|}
\hline Category & Walk kernel & Tree kernel & $\operatorname{SPM}(200)$ & SC-SPM(256) & LLC-SPM(256) & OB-SPM(LR1) & $\operatorname{SPM}(400)$ & SC-SPM(512) \\
\hline Airport & $0.882 \pm 0.023$ & $0.901 \pm 0.032$ & $0.723 \pm 0.017$ & $0.721 \pm 0.026$ & $0.723 \pm 0.017$ & $0.799 \pm 0.021$ & $0.811 \pm 0.043$ & $0.843 \pm 0.021$ \\
\hline Commer. & $0.545 \pm 0.034$ & $0.532 \pm 0.012$ & $0.441 \pm 0.023$ & $0.443 \pm 0.031$ & $0.334 \pm 0.027$ & $0.517 \pm 0.036$ & $0.521 \pm 0.022$ & $0.456 \pm 0.012$ \\
\hline Indust. & $0.642 \pm 0.021$ & $0.611 \pm 0.032$ & $0.521 \pm 0.021$ & $0.499 \pm 0.041$ & $0.413 \pm 0.015$ & $0.512 \pm 0.056$ & $0.454 \pm 0.033$ & $0.576 \pm 0.018$ \\
\hline Inter. & $0.645 \pm 0.067$ & $0.685 \pm 0.011$ & $0.611 \pm 0.018$ & $0.643 \pm 0.023$ & $0.322 \pm 0.031$ & $0.675 \pm 0.034$ & $0.674 \pm 0.026$ & $0.634 \pm 0.011$ \\
\hline Park. & $0.523 \pm 0.039$ & $0.487 \pm 0.017$ & $0.443 \pm 0.011$ & $0.512 \pm 0.037$ & $0.412 \pm 0.021$ & $0.536 \pm 0.012$ & $0.512 \pm 0.057$ & $0.496 \pm 0.025$ \\
\hline Railway & $0.556 \pm 0.076$ & $0.578 \pm 0.056$ & $0.502 \pm 0.032$ & $0.511 \pm 0.022$ & $0.521 \pm 0.033$ & $0.514 \pm 0.013$ & $0.521 \pm 0.038$ & $0.596 \pm 0.052$ \\
\hline Seaport & $0.859 \pm 0.051$ & $0.843 \pm 0.036$ & $0.774 \pm 0.021$ & $0.745 \pm 0.034$ & $0.721 \pm 0.034$ & $0.766 \pm 0.016$ & $0.632 \pm 0.043$ & $0.814 \pm 0.009$ \\
\hline Soccer & $0.646 \pm 0.021$ & $0.655 \pm 0.006$ & $0.576 \pm 0.021$ & $0.589 \pm 0.023$ & $0.578 \pm 0.023$ & $0.568 \pm 0.032$ & $0.521 \pm 0.045$ & $0.624 \pm 0.032$ \\
\hline Temple & $0.503 \pm 0.029$ & $0.454 \pm 0.031$ & $0.521 \pm 0.042$ & $0.567 \pm 0.038$ & $0.511 \pm 0.031$ & $0.603 \pm 0.021$ & $0.534 \pm 0.024$ & $0.565 \pm 0.045$ \\
\hline Univer. & $0.241 \pm 0.045$ & $0.265 \pm 0.009$ & $0.289 \pm 0.017$ & $0.301 \pm 0.021$ & $0.223 \pm 0.044$ & $0.304 \pm 0.041$ & $0.498 \pm 0.03$ & $0.321 \pm 0.012$ \\
\hline Average & $0.524 \pm 0.041$ & $0.601 \pm 0.024$ & $0.540 \pm 0.022$ & $0.553 \pm 0.030$ & $0.4770 \pm 0.033$ & $0.579 \pm 0.028$ & $0.568 \pm 0.037$ & $0.593 \pm 0.024$ \\
\hline Category & LLC-SPM (512) & OB-SPM (LRG) & $\operatorname{SPM}(800)$ & SC-SPM(1024) & LLC-SPM(1024) & OB-SPM(LRG1) & $\mathrm{SPM}(\mathrm{HC})$ & SC-SPM(HC) \\
\hline Airport & $0.801 \pm 0.021$ & $0.889 \pm 0.035$ & $0.799 \pm 0.033$ & $0.912 \pm 0.015$ & $0.899 \pm 0.019$ & $0.872 \pm 0.051$ & $0.813 \pm 0.045$ & $0.916 \pm 0.023$ \\
\hline Commer. & $0.567 \pm 0.034$ & $0.565 \pm 0.032$ & $0.512 \pm 0.032$ & $0.601 \pm 0.034$ & $0.521 \pm 0.021$ & $0.617 \pm 0.034$ & $0.519 \pm 0.043$ & $0.584 \pm 0.042$ \\
\hline Indust. & $0.521 \pm 0.025$ & $0.613 \pm 0.013$ & $0.585 \pm 0.043$ & $0.557 \pm 0.032$ & $0.593 \pm 0.019$ & $0.576 \pm 0.054$ & $0.598 \pm 0.058$ & $0.564 \pm 0.039$ \\
\hline Inter. & $0.766 \pm 0.036$ & $0.705 \pm 0.015$ & $0.644 \pm 0.022$ & $0.788 \pm 0.014$ & $0.622 \pm 0.035$ & $0.676 \pm 0.013$ & $0.668 \pm 0.041$ & $0.791 \pm 0.019$ \\
\hline Park. & $0.489 \pm 0.032$ & $0.486 \pm 0.016$ & $0.503 \pm 0.043$ & $0.489 \pm 0.043$ & $0.489 \pm 0.055$ & $0.512 \pm 0.009$ & $0.511 \pm 0.057$ & $0.487 \pm 0.025$ \\
\hline Railway & $0.553 \pm 0.042$ & $0.532 \pm 0.053$ & $0.602 \pm 0.017$ & $0.601 \pm 0.037$ & $0.599 \pm 0.009$ & $0.589 \pm 0.010$ & $0.614 \pm 0.026$ & $0.609 \pm 0.044$ \\
\hline Seaport & $0.751 \pm 0.036$ & $0.779 \pm 0.045$ & $0.815 \pm 0.031$ & $0.745 \pm 0.034$ & $0.798 \pm 0.032$ & $0.811 \pm 0.013$ & $0.822 \pm 0.039$ & $0.751 \pm 0.039$ \\
\hline Soccer & $0.625 \pm 0.026$ & $0.646 \pm 0.014$ & $0.634 \pm 0.028$ & $0.689 \pm 0.036$ & $0.655 \pm 0.014$ & $0.668 \pm 0.043$ & $0.643 \pm 0.037$ & $0.693 \pm 0.045$ \\
\hline Temple & $0.567 \pm 0.024$ & $0.587 \pm 0.027$ & $0.577 \pm 0.041$ & $0.689 \pm 0.027$ & $0.556 \pm 0.032$ & $0.612 \pm 0.025$ & $0.587 \pm 0.046$ & $0.649 \pm 0.034$ \\
\hline Univer. & $0.409 \pm 0.042$ & $0.389 \pm 0.018$ & $0.311 \pm 0.013$ & $0.582 \pm 0.035$ & $0.281 \pm 0.042$ & $0.304 \pm 0.011$ & $0.324 \pm 0.031$ & $0.537 \pm 0.033$ \\
\hline Average & $0.605 \pm 0.032$ & $0.620 \pm 0.027$ & $0.606 \pm 0.029$ & $0.654 \pm 0.033$ & $0.600 \pm 0.027$ & $0.636 \pm 0.025$ & $0.610 \pm 0.042$ & $0.658 \pm 0.032$ \\
\hline Category & LLC-SPM(HC) & Our proposed method & & & & & & \\
\hline Airport & $0.904 \pm 0.031$ & $0.864 \pm 0.051$ & & & & & & \\
\hline Commer. & $0.534 \pm 0.029$ & $0.677 \pm 0.024$ & & & & & & \\
\hline Indust. & $0.598 \pm 0.023$ & $0.555 \pm 0.034$ & & & & & & \\
\hline Inter. & $0.634 \pm 0.046$ & $0.812 \pm 0.021$ & & & & & & \\
\hline Park. & $0.493 \pm 0.064$ & $0.501 \pm 0.061$ & & & & & & \\
\hline Railway & $0.604 \pm 0.005$ & $0.606 \pm 0.033$ & & & & & & \\
\hline Seaport & $0.803 \pm 0.046$ & $0.771 \pm 0.025$ & & & & & & \\
\hline Soccer & $0.659 \pm 0.026$ & $0.663 \pm 0.065$ & & & & & & \\
\hline Temple & $0.574 \pm 0.041$ & $0.665 \pm 0.019$ & & & & & & \\
\hline Univer. & $0.287 \pm 0.049$ & $0.551 \pm 0.034$ & & & & & & \\
\hline Average & $0.609 \pm 0.036$ & $0.667 \pm 0.037$ & & & & & & \\
\hline
\end{tabular}


Table 2: Recognition accuracy under different segmentation schemes

\begin{tabular}{|c|c|c|c|c|}
\hline Category & Bench. & Defic. & Overly & Mulit. \\
\hline Intersection & 0.8 & 0.3 & 0.8 & 0.8 \\
\hline Marine & 0.4 & 0.8 & 0.8 & 0.9 \\
\hline Parking & 0.9 & 0.5 & 0.6 & 0.6 \\
\hline Residental & 0.5 & 0.7 & 0.6 & 0.7 \\
\hline School & 0.6 & 0.3 & 0.3 & 0.6 \\
\hline Average rate & 0.64 & 0.54 & 0.62 & 0.72 \\
\hline Total topology \# & 73 & 125 & 177 & 143 \\
\hline Selected structure \# & 8 & 8 & 8 & 8 \\
\hline Average RAG edge \# & 37 & 26 & 57 & 41 \\
\hline Average RAG vertex \# & 19 & 16 & 31 & 19 \\
\hline
\end{tabular}

\subsection{Discussion on different parameter settings}

We notice that the influence of segmentation operation in the RCG construction is unnegligible. To evaluate the performance under different segmentation settings (i.e., the number of singly connected regions), we perform aerial image recognition on the LHI data set, since the off-the-shelf segmentation benchmark is suitable to make a fair comparison.

Different segmentation settings are employed in our evaluation, i.e., deficient segmentation and over segmentation. The MSD values of each aerial image corresponding to different segmentation settings are computed. We observed that the benchmark segmentation setting achieves the largest MSD value 6.3, while the deficient segmentation and over segmentation gain 4.9 and 5.7, respectively. Comparatively, more regions are obtained in overly segmentations, which means it is rarer for one region to span several objects. Therefore, when building an RCG by overly segmented regions, fewer discriminative objects are neglected. Further, it is unavoidable that the unsupervised clustering is less accurate than the benchmark segmentation.

We compare the categorization accuracy under the benchmark segmentation, the over segmentation and the deficient segmentation. As shown in Table 2 , over segmen- 
tation obtains $2 \%$ lower accuracy than that of the benchmark segmentation on average. Deficient segmentation performs worse than over segmentation by providing the lowest accuracy. The overall recognition result is consistent to what the MSD reflects.

In the structure selection stage, both the threshold of MSD and MSC influence

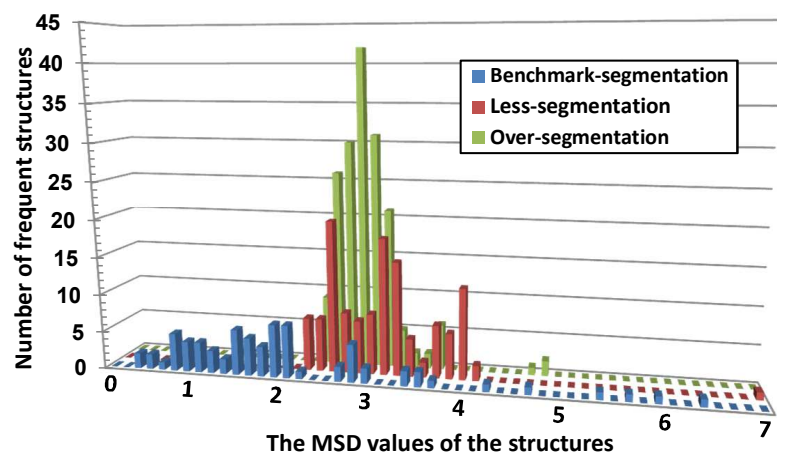

Figure 6: The discrimination of the frequent structures under different segmentation schemes.

the obtained structures. Toward an easy parameter tuning process, we set the threshold of MSD to a small value, which allows a large number of candidate structures to be qualified. Then, we tune of threshold of MSC to carefully remove those redundant structures. As shown in Fig. 7 we set the threshold of MSD to 0.1 and tune the threshold of MSC. It is observed that the categorization accuracy increases and then becomes the threshold of MSC reaches 0.65. Thus, we set the thresholds of MSD and MSC to 0.1 and 0.65 in our implementation.

\subsection{The compilation of our aerial image data set}

We compiled our data set by searching aerial images from the Google Earth. The whole data set contains 2,096 aerial images from ten categories. Since the aerial images from cities are usually clearer than those from the remote areas, we collected most of our images from metropolis, such as New York, Tokyo and Beijing. Due to the various difficulties to crawl images from different categories, the number of images in each category varies are detailed in Table 3. 


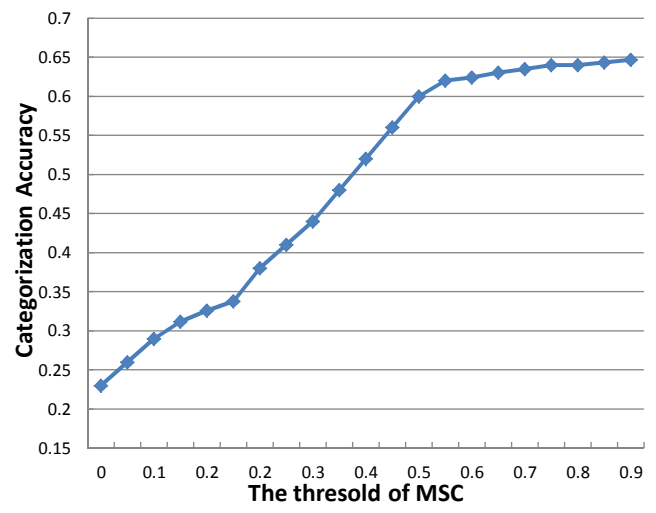

Figure 7: The categorization performance under different MSC thresholds.

Table 3: The Number of images in each category (Air.means airport, Rail. railway, Comme. commercial, Inter. intersection, Temp. template, Univ. university)

\begin{tabular}{|l|c|c|c|c|c|}
\hline Categroy & Air. & Comme. & Industrial & Inter. & Park \\
\hline Number & 306 & 262 & 206 & 302 & 129 \\
\hline Categroy & Rail. & Seaport & Soccer & Temp. & Univ. \\
\hline Number & 115 & 126 & 128 & 218 & 305 \\
\hline
\end{tabular}

\section{Conclusions}

Aerial image categorization is an important component in artificial intelligence and remote sensing [33, 34]. In this paper, a new geometric discriminative feature is proposed for aerial image recognition. Both the local features and their geometric property are taken into account to describe an aerial image. A region connected graph (RCG) is defined to encode the geometric property and the color intensity of an aerial image. Then, the frequent structures are mined statistically from the training RCGs. The refined structures are further selected from the frequent structures toward being highly discriminative and low redundant. Given a new aerial image, its geometric discriminative features are extracted guided by the refined structures, They are further quantized into a vector for SVM [24] classification. We evaluated the effectiveness of our approach on both the public and our own data sets. 


\section{Appendix}

Ideally, we want a perfect segmentation algorithm with two merits: First, each segmented region represents a semantic object/component. Second, the segmentation algorithm is parameter-free. Thus, we can apply it to segment thousands of training images once for all, without human-interactive parameter tuning. Unfortunately, for the first merit, the high-level features in those semantics-exploited segmentation methods are usually designed manually and data set dependent, which is not consistent with the fully-automated and data set independent framework of the proposed method; besides, to learn semantics, semantics-exploited segmentation methods typically require well-annotated training images, however, the large number of training aerial images used in our experiment are online crawled and human annotation is laborious. For the second merit, those semantic-exploited segmentation methods are usually complicated and there are several important user-controlled parameters. Therefore, we can only use those data-driven segmentation methods, where no semantics are explored and typically contain one tuning parameter. Those well-known data-driven segmentation algorithms can be divided into two groups. The first group algorithms need the number of segmented regions as input, such as k-means and normalized cut; however, there is no uniform segmented region number on different images because different images usually contain different number of components. The second group algorithms require some tolerance bound as input, such as the similarity tolerance between spatially neighboring segmented regions. Compared with segmented region number, we empirically found that the tolerance bound is more flexible to tune. Therefore, in our approach, we chose the second group data-driven segmentation methods. After some experimental comparison, we found that the unsupervised fuzzy clustering 2 outperforms several tolerance bound-based segmentation algorithms, such as graph-based segmentation 3 . Thus, we choose unsupervised fuzzy clustering in our approach.

\footnotetext{
${ }^{2}$ Matlab codes: https://mywebspace.wisc.edu/pwang6/personal/

${ }^{3} \mathrm{C}++$ codes: http://www.cs.brown.edu/ pff/segment/
} 


\section{References}

[1] X. Yuan, H. Zhu, S. Yang, "A Robust Framework For Eigenspace Image Reconstruction," IEEE Workshop on Appl. of Comp. Vis., pp. 54-59, 2005.

[2] H. Blum, "Biological shape and visual science", Journal of Theoretical Biology, pages 205-287, 1973.

[3] J. Porway, K. Wang, B. Yao, S.C. Zhu, "Scale-invariant shape features for recognition of object categories", in Proc. IEEE Int. Comp. Vis., pp. 90-96, 2004.

[4] M. A. Maloof, P. Langley, T. O. Binford, R. Nevatia, S. Sage, "Improved Rooftop Detection in Aerial Images with Machine Learning", Machine Learning, pages 157-191, 2003.

[5] T. Zhao, R. Nevatia, "Car detection in low resolution aerial image", in Proc. IEEE Int. Comp. Vis., 2001.

[6] L. Zhang, Y. Gao, Y. Xia, Q. Dai, X. Li, A Fine-Grained Image Categorization System by Cellet-Encoded Spatial Pyramid Modeling, IEEE Transcations on Industrial Electronics (T-IE), 2014 (accepted).

[7] L. Zhang, Y. Gao, C. Hong, Y. Feng, J. Zhu, D. Cai, Feature Correlation Hypergraph: Exploiting High-order Potentials for Multimodal Recognition, IEEE Transcations on Cybernetics (T-CYB), 2013 (accepted).

[8] L. Zhang, Y. Gao, R. Ji, L. Ke, J. Shen, Representative Discovery of Structure Cues for Weakly-Supervised Image Segmentation, IEEE Transcations on Multimedia (TMM), 16(2): 470-479, 2014.

[9] L. Zhang, M. Song, Y. Yang, Q. Zhao, Z. Chen, N. Sebe, Weakly Supervised Photo Cropping, IEEE Transcations on Multimedia (T-MM), 16(1): 94-107, 2014.

[10] L. Zhang, M. Song, Z. Liu, X. Liu, J. Bu, C. Chen, Probabilistic Graphlet Cut: Exploring Spatial Structure Cue for Weakly Supervised Image Segmentation, IEEE Computer Vision and Pattern Recognition (CVPR), pages: 1908-1915, 2013. 
[11] H. Moissinac, H. Maitre, I. Bloch, "Urban aerial image understanding using symbolic data", In in Proc. SPIE Image and signal proce. for remote sensing, 1994.

[12] A. C. Berg, F. Grabler, J. Malik, "Parsing images of architectural scenes", in Proc. IEEE Int. Comp. Vis., pp. 1-8, 2007.

[13] J. Porway, K. Wang, B. Yao, S.C. Zhu, "A hierarchical and contextual model for aerial image understanding", in Proc. IEEE Int. Comp. Vis., pp. 1-8, 2008.

[14] S. Lazebnik, C. Schmid, J. Ponce, "Beyond bags of features: spatial pyramid matching for recognizing natural scene categories", in Proc. IEEE Int. Comp. Vis., pp. 2169-2178, 2006.

[15] N. Cristianini, B. Scholkopf. "Using spin images for efficient object recognition in cluttered 3D scenes", IEEE Trans. on Pattern Analysis and Mach. Intell., vol. 21, no. 5, pp. 433-449, 1999.

[16] Z. Harchaoui, F. Bach, "Image classification with segmentation graph kernels", in Proc. IEEE Int. Comp. Vis., pp. 1-8, 2007.

[17] R. Gonzalez, R. Woods, S. Eddins, "Digital Image Processing Using Matlab". Prentice Hall, Dec 26, 2003.

[18] S. Jia, Z. Zhu, L. Shen, Q. Li, "A two-stage feature selection framework for hyperspectral image classification using few labeled samples", IEEE J. Sel. Topics Appl. Earth Observ. Remote Sens., vol. 7, no. 4, pp. 1023-1035, 2014.

[19] C. Chen, W. Li, E. W. Trame, M. Cui, S. Prasad, J. E. Fowler, "Spectral-spatial preprocessing using multihypothesis prediction for noise-robust hyperspectral image classification”, IEEE J. Sel. Topics Appl. Earth Observ. Remote Sens., vol. 7, no. 4, pp. 1047-1059, 2014.

[20] N. Sherashidze, S.V.N. Vishwanathan, T.H. Petri, K. Mehlhorn, K.M. Borgwardt, "Efficient graphlet kernels for large graph comparison", Int. Conf. on Artif. Intell. and Stat., pp. 488-495, 2009. 
[21] M. Kuramochi, G. Karypis. "An efficient algorithm for discovering frequent subgraphs", IEEE Trans. Knowledge and Data Eng., vol. 16, no. 9, pp. 1038-1051, 2004.

[22] J. R. Ullmann, "An algorithm for subgraph isomorphism", Journal of the ACM, vol. 23, no. 1, pp. 31-42, 1976.

[23] L. Zhang, M. Song, N. Li, J. Bu, C. Chen, "Feature selection for fast speech emotion recognition”, ACM Multimedia, pp. 753-756, 2009.

[24] N. Cristianini, B. Scholkopf, "Support vector machines and kernel methods: the new generation of learning machines", AI Magzine, vol. 23, no. 3, pp. 31-41, 2002.

[25] Y. Li, S. Gong, H. Liddell, "Kernel discriminant analysis", ACM Trans. Program. Lang. Syst., vol. 15, no. 5, pp. 745-770, 1998.

[26] H.-M. Chen, C. Lin, S.-Y. Chen, C.-H. Wen, C.-C. Chen, Y.-C. Ouyang, C.-I Chang, "PPI-SVM-Iterative FLDA Approach to Unsupervised Multispectral Image Classification”, IEEE J. Sel. Topics Appl. Earth Observ. Remote Sens., vol. 6, no. 4, pp. 1834-1842, 2013.

[27] L. Zhang, Y. Gao, R. Ji, Q. Dai, X. Li, Actively Learning Human Gaze Shifting Paths for Photo Cropping, IEEE Transcations on Image Processing (T-IP), 23(5), pages: 2235-2245, 2014.

[28] L. Zhang, Y. Gao, R. Zimmermann, Q. Tian, X. Li, Fusion of Multi-Channel Local and Global Structural Cues for Photo Aesthetics Evaluation, IEEE Transcations on Image Processing (T-IP), 23(3): 1419-1429, 2014.

[29] L. Zhang, Y. Yang, C. Wang, X. Li, A Probabilistic Associative Model for Segmenting Weakly- Supervised Images, IEEE Transcations on Image Processing (TIP), 2014 (accepted).

[30] L. Zhang, R. Ji, Y. Xia, X. Li, Learning a Probabilistic Topology Discovering Model for Scene Categorization, IEEE Transcations on Neural Networks and Learning Systems (T-NNLS), 2014 (accepted). 
[31] X. Liu, M. Song, D. Tao, L. Zhang, J. Bu, C. Chen, Learning to Track Multiple Objects, IEEE Transcations on Neural Networks and Learning Systems (T-NNLS), 2014 (accepted).

[32] L. Zhang, Y. Gao, Y. Xia, R. ji, X. Li, Spatial-Aware Object-Level Saliency Prediction by Learning Graphlet Hierarchies, IEEE Transcations on Industrial Electronics (T-IE), 2014 (accepted).

[33] B. Luo, S. Jiang, L. Zhang, "Indexing of Remote Sensing Images With Different Resolutions by Multiple Features”, IEEE J. Sel. Topics Appl. Earth Observ. Remote Sens., vol. 6, no. 4, pp. 1899-1912, 2013.

[34] A. Makarau, G. Palubinskas, P. Reinartz, "Alphabet-Based Multisensory Data Fusion and Classification Using Factor Graphs", IEEE J. Sel. Topics Appl. Earth Observ. Remote Sens., vol. 6, no. 2, pp. 969-990, 2013.

[35] J. Shi, J. Malik, "Normalized cuts and image segmentation", IEEE Trans. on Pattern Analysis and Mach. Intell., vol. 22, no. 8, pp. 888-905, 2000.

[36] B. Yao, X. Yang, S.-C. Zhu, "Introduction to a large scale general purpose ground truth dataset: methodology, annotation tool, and benchmarks", EMMCVPR, 2007.

[37] X. Xiong, K. L. Chan, "Towards an unsupervised optimal fuzzy clustering algorithm for image database organization", in Proc. IEEE Conf. Pattern Recognit, pp. 3909, 2000.

[38] L. Zhang, Y. Han, Y. Yang, M. Song, S. Yan, Q. Tian, Discovering Discrminative Graphlets for Aerial Image Categories Recognition, IEEE Transcations on Image Processing (T-IP), 22(12):5071-5084, 2013.

[39] L. Zhang, M. Song, Q. Zhao, X. Liu, J. Bu, C. Chen, Probabilistic Graphlet Transfer for Photo Cropping, IEEE Transcations on Image Processing (T-IP), 21(5): 2887-2897, 2013.

[40] T. H. Cormen, C. E. Leiserson, R. L. Rivest, C. Stein, "Introduction to Algorithms", MIT Press and McGraw-Hill, pp. 540-549, 2001. 
[41] J. Wang, J. Yang, K. Yu, F. Lv, T. Huang, Y. Gong, "Locality-constrained linear voding for image classification", in Proc. IEEE Conf. Comput. Vis. Pattern Recognit., pp. 3360-3367, 2010.

[42] J. Yang, K. Yu, Y. Gong, T. Huang, "Linear spatial pyramid matching using sparse coding for image classification", in Proc. IEEE Conf. Comput. Vis. Pattern Recognit., pp. 2169-2178, 2009.

[43] L.-J. Li, H. Su, E. P. Xing, F.-F. Li, Object Bank: A High-Level Image Representation for Scene Classification and Semantic Feature Sparsification, in Proc. Adv. Neural Inf. Process. Syst, pp. 1378-1386, 2010. 